\title{
Atenção Pré-Natal no Município de Quixadá-CE segundo indicadores de processo do SISPRENATAL
}

\author{
PRENATAL CARE IN QUIXADÁ-CE ACCORDING TO SISPRENATAL'S PROCESS INDICATORS
}

\author{
ATENCIÓN PRENATAL EN EL MUNICIPIO DE QUIXADÁ-CE SEGÚN \\ INDICADORES DE PROCESO DEL SISPRENATAL
}

\section{Gisele Ribeiro Grangeiro', Maria Albertina Rocha Diógenes²,Escolástica Rejane Ferreira Moura³}

\begin{abstract}
RESUMO
Pesquisa descritiva, documental, que objetivou analisar os indicadores de processo do Sistema de Informação do Prénatal (SISPRENATAL), em Quixadá-CE. Estudaram-se 1.544 cadastros de gestantes no período de 2001 a 2004. Os dados foram coletados de fevereiro a abril de 2005 no SISPRENATAL, implantado no setor de Epidemiologia da Secretaria Municipal de Saúde. Os resultados foram apresentados em tabela única e analisados à luz da literatura atual e experiência acumulada das autoras. Verificou-se percentual crescente de gestantes com indicador de, no mínimo, seis consultas, todos os exames básicos, teste anti-HIV, imunização antitetânica e consulta puerperal de 2001 a 2004, ou seja, zero, 2,6, 5,68 e 21,11\%, respectivamente. É necessário, pois, uma melhora na utilização do Sistema, assim como a leitura sistemática dos indicadores de processo, no sentido de obter subsídios para a melhoria da qualidade da assistência prénatal.
\end{abstract}

\section{DESCRITORES}

Avaliação de Programas e

Projetos de Saúde.

Parto humanizado.

Cuidado pré-natal.

\begin{abstract}
This is a descriptive, documental survey aimed at analyzing the process indicators of the Prenatal Information System (Sisprenatal) in the municipality of Quixadá, State of Ceará. The records of 1,544 pregnant women from 2001 through 2004 were studied. Data were collected from February through April, 2005 in Sisprenatal, located in the Epidemiology sector of the local Health Secretary. The results were presented in a single chart containing absolute and relative frequency. They were analyzed in the light of current literature and the authors' experience. In the period was verified a growing percentage of pregnant women with indicators of at least six appointments, all the basic exams, antiHIV test, antitetanic immunization and puerperal consultation, from zero percent (2001), to $2.6 \%$ (2002), to $5.68 \%$ (2003) to $21.11 \%(2004)$. Thus it is necessary to improve the use of the system, as well as the systematic reading of the process indicators, in order to obtain subsidies for the enhancement of the quality of prenatal assistance.
\end{abstract}

\section{KEY WORDS}

Program evaluation.

Humanizing delivery.

Prenatal care.

\begin{abstract}
RESUMEN
En esta Investigación descriptiva, documental se tuvo como objetivo analizar los indicadores de proceso del Sistema de Información del Prenatal (SISPRENATAL), el el municipio de Quixadá-CE. Fueron estudiados 1.544 registros de mujeres embarazadas en el período de 2001 a 2004. Los datos fueron recolectados de febrero a abril del 2005 en el SISPRENATAL, en el sector de Epidemiología de la Secretaría Municipal de Salud. Los resultados fueron presentados sólo en una tabla y analizados a la luz de la literatura actual y también a partir de las experiencias de las autoras. Se verificó el porcentaje creciente de mujeres embarazadas con indicador mínimo de seis consultas, todos los exámenes básicos, test anti-VIH, inmunización antitetánica y consulta puerperal de 2001 a 2004, es decir, cero, 2,6, 5,68 e $21,11 \%$, respectivamente. Es necesario, pues, una mejor utilización del Sistema, y también una lectura sistemática de los indicadores de proceso para obtener soporte para la mejoría de la calidad de la asistencia prenatal.
\end{abstract}

\section{DESCRIPTORES \\ Evaluación de Programas y \\ Proyectos de Salud. \\ Parto humanizado. \\ Atención prenatal.}




\section{INTRODUCÃ̃}

A assistência pré-natal compreende um conjunto de cuidados e procedimentos que visa preservar a saúde da gestante e do concepto, assegurando a profilaxia e a detecção precoce das complicações próprias da gestação e o tratamento adequado de doenças maternas pré-existentes. Também deve incluir orientações sobre hábitos saudáveis de vida e as modificações resultantes da gravidez, bem como o preparo da gestante para o parto e o puerpério ${ }^{(1)}$.

Apesar de ser uma prioridade entre as ações praticadas pelos serviços de saúde, marcadamente a partir de 1984, com a implantação do Programa de Assistência Integral à Saúde da Mulher (PAISM) ${ }^{(2)}$, persiste a dificuldade de acesso a essa assistência em algumas regiões. A baixa qualidade da atenção prestada à mulher, no ciclo graví-dico puerperal, resulta em elevadas taxas de morbidade e mortalidade materna e perinatal nas diversas esferas. Como recomendação do PAISM, os serviços já deveriam desenvolver discussão permanente com a população adstrita, especialmente com as mulheres, sobre a importância da assistência pré-natal, de forma a obter adesão das gestantes ao serviço de pré-natal ainda no primeiro trimestre de gravidez. Havia preocupação com a disponibilização de recursos humanos treinados, atendendo em área física adequada e equipada com instrumental mínimo, apoio labo-ratorial e instrumentos de registro, proces-samento e análise de dados, estruturação de um sistema de referência e contra-referência a permitir assistência às gestantes nos três níveis de complexidade do sistema de saúde, e avaliação permanente das ações desenvolvidas ${ }^{(3)}$.

Em 1994, o município de Quixadá-CE destacou seu pioneirismo na estratégia de levar os serviços de saúde para mais próximo de onde as pessoas viviam, reordenando o modelo assistencial em saúde e contribuindo com experiência exitosa para a consolidação do Sistema Único de Saúde (SUS) e a implantação do Programa Saúde da Família (PSF). Neste contexto, a atenção ao pré-natal avançou, consideravelmente, em cobertura, uma vez que foram implantadas 17 Equipes de Saúde da Família (ESF), sendo oito em zona urbana e nove em zona rural, número que se mantém até os dias atuais. Com uma população de 74.791 habitantes, essas 17 ESF correspondem a uma cobertura de $98 \%$ da população do município. Nas unidades básicas de saúde são desenvolvidas atividades referentes à imunização, prevenção do câncer de colo do útero e detecção precoce do câncer de mama, assistência pré-natal, acompanhamento ao hiper-tenso e diabético, atendimento à criança, ao adulto e ao idoso e coleta sistemática de exames laboratoriais, que são encaminhados para análise no Laboratório Central do Município. O Hospital Maternidade local é a referência obstétrica e pediátrica para o PHPN (Programa de Humanização de Pré-Natal e Nascimento), possuindo Unidade de Terapia Intensiva (UTI) Neonatal. Estudo realizado na Região de Saúde de Quixadá afirmou que oito óbitos maternos foram notificados de 1999 a 2003, no entanto, o município de Quixadá não registrou nenhum óbito no mesmo período ${ }^{(4)}$.

O Brasil registrou, em 1998, 64,8 óbitos maternos para 100.000 nascidos vivos, sendo a primeira causa de morte a síndrome hipertensiva ${ }^{(3)}$. O Ceará registrou, no mesmo ano, 93,3 óbitos maternos para 100.000 nascidos vivos, ou seja, dados superiores à média nacional, mantendo, também, como causa principal, a hipertensão induzida pela gestação (HIG). No período de 2000 a 2002, as quatro principais causas da morte materna, no Estado, foram a síndrome hipertensiva (42,8\%), as síndromes hemorrá-gicas (27,9\%), as infecções puerperais $(16,1 \%)$ e as complicações do aborto $(13 \%)$, causas essencialmente prevení-veis, se garantida a assistência pré-natal de qualidade (SES-CE, 2003) ${ }^{(5)}$. Um outro indicador preocupante diz respeito à elevada incidência de sífilis congênita, estimada em 12 casos por 1000 nascidos vivos no SUS ${ }^{(5)}$.

No Brasil, o número de consultas de prénatal por mulher que realiza o parto no SUS vem aumentando, passando de 1,2 consultas por parto em 1995, para 5,1 consultas por parto em $2003^{(6)}$. Assim, apesar da cobertura pré-natal ter melhorado consideravelmente, inclusive após a implantação das equipes do PSF, estratégia iniciada em 1994, com os princípios e desafios apontados à época da proposição do PAISM, os índices elevados de óbitos maternos persistem, pondo em pauta a qualidade das consultas do pré-natal.

Reconhecendo, pois, a necessidade imposta de estabelecer mecanismos que viabilizassem a melhoria da qualidade do acompanhamento pré-natal, o Ministério da Saúde instituiu, em $1^{\circ}$. de junho de 2000 , através da Portaria GM/MS n 569/GM 2002, o Programa de Humanização no Pré-Natal e Nascimento (PHPN), que apresenta em sua formulação os objetivos de reduzir as altas taxas de morbidade e mortalidade materna e perinatal, ampliar o acesso ao pré-natal, estabelecer critérios para qualificar as consultas e promover o vínculo entre a assistência ambulatorial e o parto, articulando três componentes: I - Incentivo à assistência pré-natal no âmbito do Sistema Único de Saúde (SUS); II - Organização, regulação e investimentos na assistência obstétrica e neonatal na área hospitalar; e III - Instituição de nova sistemática de pagamento da assistência ao parto ${ }^{(7)}$.

Essa proposta fez com que cada município retomasse a discussão sobre a atenção à gestação, parto e puerpério em seu território, detectando diversas lacunas na capacidade instalada, marcadamente com relação à oferta dos exames laboratoriais, atendimento especializado para as gestantes de risco e a referência para o parto. 
Em relação ao acompanhamento das gestantes, a Portaria estabelece, em seu Anexo I, que os serviços que aderirem ao programa deverão realizar, no mínimo:

- a primeira consulta pré-natal até o 40 mês da gestação; seis consultas durante o pré-natal, sendo, preferencialmente, uma no primeiro trimestre, duas no segundo e três no terceiro trimestre da gestação; uma consulta no puerpério até 42 dias após o nascimento; os exames laboratoriais mínimos (ABO-Rh, na primeira consulta; VDRL, um exame na primeira consulta e um na trigésima semana da gestação; urina tipo I, um exame na primeira consulta; glicemia de jejum, um exame na primeira consulta e um na trigésima semana da gestação; $\mathrm{Hb} / \mathrm{Ht}$, na primeira consulta e testagem anti-HIV, nos municípios com população acima de 50.000 hab.); aplicação de vacina antitetânica; atividades educativas; classificação de risco gestacional; e referência à gestação de alto risco.

Cada município cadastrado no PHPN recebe um incentivo de $R \$ 10,00$ por gestante inscrita até os 120 dias de idade gestacional, $R \$ 40,00$ relativos à conclusão do acompanhamento, devendo cumprir com todos os critérios estabelecidos pelo Programa e $\mathrm{R} \$ 40,00$ destinam-se ao hospital que realizar o parto, desde que o mesmo integre a rede de referência para este tipo de atendimento, contida nos Termos de Adesão do município. Assim, para a avaliação do PHPN, foi criado o Sistema de Informação do Pré-natal (SISPRENATAL), com os objetivos de possibilitar o monitoramento do Programa pelos gestores do SUS, a partir de informação mensal efetuada pelas equipes das unidades básicas de saúde dos municípios cadastrados no Programa, e acompanhar o cumprimento dos critérios para o pagamento dos incentivos ${ }^{(8)}$.

O sistema gera indicadores de processo que incluem: percentual de gestantes que se inscrevera no programa e realizaram a primeira consulta até o 40 mês de gestação, em relação à população-alvo (número de gestantes existentes ou estimadas pelo número de nascidos vivos do município); percentual de gestantes inscritas que realizaram seis consultas de pré-natal; percentual de gestantes inscritas que realizaram seis consultas de pré-natal e os exames laboratoriais preconizados; percentual de gestantes inscritas que realizaram seis consultas de prénatal, a consulta de puerpério e os exames laboratoriais; percentual de gestantes inscritas que receberam dose imunizante da vacina antitetânica, no mínimo duas doses, durante o pré-natal; e percentual de gestantes inscritas que realizaram seis consultas no pré-natal, a consulta de puerpério e os exames básicos, o teste anti-HIV e a dose imunizante da vacina antitetânica e outros indicadores de resultado e de impacto.

Tendo em vista a magnitude da assistência pré-natal na perspectiva do PHPN, decidiu-se pela realização do presente estudo, que objetivou analisar os indicadores de processo da assistência pré-natal gerados no SISPRENATAL.

\section{MÉTODO}

Pesquisa descritiva, documental, abordando indicadores de processo da assistência pré-natal em Quixadá$\mathrm{CE}$, utilizando como fonte de dados o SISPRENATAL, implantado no setor de Epidemiologia da Secretaria Municipal de Saúde (SMS). A pesquisa descritiva traduz um delineamento da realidade da assistência pré-natal, descrevendo-a, analisando-a e interpretando-a $a^{(9)}$.

O referido sistema recebe informações contidas em duas fichas: a Ficha de Cadastramento da Gestante e a Ficha de Registro Diário dos Atendimentos da Gestante. A Ficha de Cadastramento da Gestante é preenchida na primeira consulta do pré-natal pelo médico ou enfermeiro que realiza o atendimento, anotando o número do cadastro no cartão da gestante. Estes profissionais também são responsáveis pelo preenchimento da Ficha de Registro Diário de Atendimento da Gestante em todas as consultas subseqüentes ${ }^{(8)}$. O SISPRENATAL elabora e disponibiliza os indicadores de processo, tomando como base de cálculo a estimativa do número de nascidos vivos naquele município no mesmo ano. Porém, para a obtenção do indicador de processo percentual de gestantes que foram inscritas no PHPN e realizaram a primeira consulta até o quarto mês é tomado como base de cálculo o número de gestantes existentes.

Foram analisados dados relativos a 1.544 gestantes cadastras no PHPN, no período de 2001 a 2004.

Os dados obtidos foram apresentados em tabela única, contendo freqüência relativa, descrevendo por meio de estatística simples os respectivos indicadores. Estes foram analisados comparativamente ano a ano e interpretados à luz da literatura atual e experiências das autoras.

O estudo seguiu as recomendações estabelecidas na Resolução n 196/96 do Ministério da Saúde, que se refere à pesquisa envolvendo seres humanos ${ }^{(10)}$.

\section{RESULTADOS}

Em 2001, foram inscritas 166 gestantes no PHPN do município estudado, correspondendo a $15,05 \%$ de cobertura; em 2002, 462 gestantes foram cadastradas, representando uma cobertura de $34,27 \%$ e, portanto, um incremento de mais de $100 \%$ com relação ao primeiro ano (2001); em 2003, 456 foram cadastradas (33,83\% de cobertura) destacando, assim, pequena redução com relação a 2002; e, em 2004, 460 foram inscritas no Programa, voltando a crescer o cadastramento em comparação ao ano anterior, ora representando $34,12 \%$ de cobertura.

Verificou-se crescimento na porcentagem de gestantes cadastradas que realizaram, no mínimo, seis consultas de pré-natal ao longo dos anos estudados. Quando foi 
incluída a variável consulta puerperal a esse indicador, as porcentagens decaíram.

O percentual de gestantes que realizara, no mínimo, seis consultas de pré-natal, associado à variável realização dos exames básicos, mostrou-se irregular ao longo dos anos, ou seja, cresce em 2002, mostra uma queda abrupta em 2003 e volta a crescer, consideravelmente, em 2004.

O percentual de gestantes que atingiu seis ou mais consultas de pré-natal, associado à variável de realização dos exames básicos e à consulta de puerpério, apresentou-se ainda mais reduzido, revelando, porém, perfil semelhante de ascensão em 2002, acentuado declínio em 2003, e revelando crescimento em 2004.
Os dados relativos à imunização da gestante demonstraram melhoria expressiva a partir de 2002, chegando, em 2004, com 90,9\% das gestantes cadastradas imunizadas.

Quando foi acrescentado aos critérios, já comentados anteriormente, a realização do exame anti-HIV, verificaram-se porcentagens ainda menores. A realização do exame pelas gestantes, em 2001, não existiu, mantendo-se níveis baixíssimos, em 2002, com 2,6\% e, 2003, com 5,68\%, obtendo uma discreta melhora, em 2004 para 21,11\%, como visto na tabela a seguir.

A Tabela 1 apresenta os indicadores de processo do SISPRENATAL referente ao período 2001-2004, com um número total de 1.544 gestantes cadastradas ao longo dos anos $(n=1.544)$.

Tabela 1 - Indicadores de processo da atenção pré-natal, SISPRENATAL - Quixadá, CE - 2001-2004

\begin{tabular}{|c|c|c|c|c|}
\hline Indicadores de processo & 2001 & 2002 & 2003 & 2004 \\
\hline $\begin{array}{l}\text { Primeira consulta até o quarto mês, em relação ao número de nascidos vivos do município } \\
\text { no período. }\end{array}$ & 15,05 & 34,27 & 33,83 & 34,12 \\
\hline No mínimo, seis consultas de pré-natal. & --- & 39,84 & 45,97 & 64,98 \\
\hline No mínimo, seis consultas de pré-natal e a consulta de puerpério. & --- & 22,08 & 32,2 & 37,5 \\
\hline No mínimo, seis consultas de pré-natal e todos os exames básicos. & --- & 21,09 & 12,1 & 52,32 \\
\hline No mínimo, seis consultas de pré-natal, a consulta de puerpério e todos os exames básicos. & --- & 16,88 & 7,95 & 22,22 \\
\hline $\begin{array}{l}\text { No mínimo, duas doses de antitetânica durante o pré-natal ou dose de reforço em mulheres já } \\
\text { imunizadas ou nenhuma dose nas mulheres com imunização completa. }\end{array}$ & --- & 63,03 & 63,88 & 90,09 \\
\hline $\begin{array}{l}\text { No mínimo, seis consultas de pré -natal, a consulta de puerpério, todos os exames básicos, o } \\
\text { teste anti-HIV e a imunização antitetânica. }\end{array}$ & --- & 2,6 & 5,68 & 21,11 \\
\hline
\end{tabular}

Fonte: Secretaria de Saúde do Município, SISPRENATAL

\section{DISCUSSÃO}

O reduzido cadastramento das gestantes em Quixadá-CE, no ano de 2001 (15,05\%), pode estar relacionado ao início da implantação do SISPRENATAL no município, estando, assim, sujeito às falhas opera-cionais do sistema, dos profissionais responsáveis pela assistência ou pela alimentação do mesmo. Esse pensamento também pode justificar a ausência de informações com relação às demais variáveis, no mesmo ano, o que levou a não implantação dos indicadores de processo do PHPN, como constatado a partir de uma leitura vertical da tabela.

Em pesquisa realizada no território nacional sobre a análise de informações do SISPRENATAL, no período 20012002, os dados apontaram baixo cadastramento de muIheres em 2001 (9,3\%), havendo um aumento em 2002 $(28 \%)^{(11)}$. A realidade, mostrada pela pesquisa mencionada, não difere da situação encontrada no Município de Quixadá, que também apresentou baixo índice em 2001, e, em 2002, houve um aumento no percentual de gestantes cadastradas, passando para $34,27 \%$.
Contudo, estes percentuais se mantiveram, praticamente, estáveis entre 2002 (34,27\%) e 2004 (34,12\%), evidenciando que os valores dos indicadores estudados ainda permanecem reduzidos, indicando necessidade de esforços para aumentar a cobertura e a captação precoce das gestantes. Há que se considerar o estabelecimento de uma avaliação sistemática no município, de maneira a proporcionar intervenções oportunas à melhoria da qualidade da atenção pré-natal.

Acredita-se que uma maior capacitação dos profissionais envolvidos, tanto no cadastramento na unidade de saúde quanto na digitação desses dados na SMS, são procedimentos fundamentais ao bom desenvolvimento do SISPRENATAL.

O início do pré-natal, o mais precoce possível, objetiva fortalecer a adesão da mulher ao acompanhamento sistemático e, assim, rastrear eventuais fatores de risco. O acompanhamento da gestante deve ter início precoce, ter cobertura universal, ser realizado de forma periódica, estar integrado com as demais ações preventivas e cu- 
rativas e observar um número mínimo de consultas ${ }^{(12)}$. Seu sucesso depende, em grande parte, do momento em que ele se inicia e do número de consultas realizadas, podendo variar de acordo com o mês de início e com as intercorrências durante a gravidez. Em estudo realizado com 520 pacientes em uma maternidade de Juiz de Fora - MG, em 2002, detectou-se que apenas $29,7 \%$ das gestantes haviam iniciado o pré-natal no primeiro trimestre, ou seja, a dificuldade de captação precoce das gestantes repete-se também em outros municípios ${ }^{(13)}$. Estudo realizado, em um Hospital Público do Rio de Janeiro, revisando 118 fichas de gestantes que realizaram consulta pré-natal de janeiro a junho de 2003, identificou $42,3 \%$ de captação precoce ao acompanhamento prénatal(14).

O percentual de gestantes que realizara, pelo menos, seis consultas no pré-natal, apesar de ter se mostrado baixo no universo pesquisado, superou a porcentagem nacional de 2002 , em $19,84 \%$, pois evidenciou-se que, em todo o País, um percentual de cerca de $20 \%$ das gestantes realizaram as seis consultas no mesmo período(11). A realização das seis consultas durante o pré-natal seria o mínimo necessário para garantir não somente o acompanhamento dos parâmetros que avaliam a evolução da gestação e a detecção de fatores de risco e agravos que poderão surgir, mas, de igual importância, estabelecer vínculo com as gestantes e sua família, no sentido de bem preparála para o parto e o puerpério, de maneira a vivenciá-los com autonomia e segurança.

No puerpério, podem ocorrer diversas alterações na saúde da mãe e do recém-nascido, principalmente se, no período gestacional, forem evidenciadas complicações ou se não houve uma boa cobertura de consultas. A consulta puerperal serve para o estabelecimento de condutas que garantam o adequado intervalo interpartal, que protege a mulher e melhora os resultados perinatais, com a orientação para a introdução de método contraceptivo, assim como a avaliação que permita detectar importantes alterações, como a anemia, estado depressivo e dificuldades relacionadas ao aleitamento materno. A inclusão da consulta puerperal, como ação extensiva e integradora do acompanhamento pré-natal, vem sendo incentivada com vigor desde a implantação do PAISM, em 1984, com a meta de se garantir uma assistência de qualidade às mulheres ${ }^{(2)}$. Todavia, percentuais baixos de gestantes estão tendo esse direito assegurado. Um estudo ${ }^{(15)}$ defende ser de extrema necessidade a realização de no mínimo uma consulta puerperal, com até 42 dias após o nascimento, conforme preconiza o Ministério da Saúde, considerando-a indispensável à detecção de intercorrências obstétricas e ginecológicas, bem como para fornecer informações relevantes sobre os cuidados que a mulher deve tomar consigo e com o recém-nascido, como orientações sobre a amamentação, contracepção e sexualidade. $\mathrm{O}$ indicador de consulta puerperal, neste estudo, demonstrou deficiência importante no registro.
Os exames laboratoriais básicos são imprescindíveis no acompanhamento pré-natal, uma vez que complementam a análise dos dados clínicos e obstétricos, favorecendo a adoção de diagnósticos e as condutas adotadas com relação aos mesmos. Neste estudo, revelou-se que somente metade das gestantes inseridas no serviço de pré-natal tem acesso aos exames. É enfatizado que, no decorrer da fase de adesão dos municípios ao PHPN,

muitos se surpreenderam ao estimar as necessidades anuais de exames laboratoriais para atender às gestantes (informação obrigatória para o termo de adesão ao PHPN), pois estas respondiam por quase toda quota de exames disponibilizados pelo SUS naquela cidade ${ }^{(16)}$.

Portanto, há uma lacuna na oferta de serviços laboratoriais que precisa de investimentos em todas as esferas de governo para ser superada.

Para avaliar adequadamente a condição vacinal da gestante, é necessário o registro de imunidade anterior à gestação atual, para assim, tomar uma conduta correta. Apesar de a vacinação antitetânica estar disponível em, praticamente, todos os serviços de saúde, ainda existem mulheres que não recebem nenhuma dose de vacina e um número importante que não é corretamente imunizado. Embora os casos de tétano neonatal tenham declinado nos últimos anos, em todas as regiões, principalmente pelo aumento da cobertura hospitalar ao parto e da vacinação sistemática de gestantes e mulheres em idade fértil, é necessário o investimento contínuo na melhor cobertura vacinal ${ }^{(3)}$. Nenhum caso de tétano neonatal é registrado em Quixadá, desde o ano 2000 até o período deste estudo. Com o aumento da imunização antitetânica no pré-natal, a partir de 2002, chegando a 90,09\% em 2004, espera-se que as equipes do PSF atinjam $100 \%$ das mulheres cadastradas e, assim, mantenha-se a ausência de casos, definitivamente, no município.

Apesar de o indicador que associa o teste anti-HIV ter mostrado aumento progressivo, ao longo do período estudado, a cobertura do exame é muito baixa. Dentre as faIhas de detecção da infecção pelo HIV durante a gestação, destacam-se a ausência ou início tardio do acompanhamento pré-natal, sem tempo para obter o resultado da sorologia para o HIV e a devida intervenção; atendimento pré-natal sem solicitação do teste anti-HIV; atendimento pré-natal com solicitação do teste anti-HIV, mas sem resultado do exame laboratorial em tempo hábil ou extravio do exame ${ }^{(17)}$. Essas afirmativas corroboram com a realidade do município estudado, acrescentando-se o fato de que o exame anti-HIV é ofertado à gestante, contudo, ela não tem obrigatoriedade em fazê-lo.

Em pesquisa realizada com 138 grávidas infectadas pelo HIV, no município de Fortaleza-CE, no período de 1999 à 2001, foi identificado, após o cruzamento de todos os bancos de dados empregados no estudo, que 35,5\% já sabiam do seu status sorológico antes da gestação, 48,6\% souberam durante o pré-natal, ao passo que $4,3 \%$ desco- 
briram-se infectadas pelo HIV durante o parto e $11,6 \%$ após o parto ou pelo diagnóstico da criança. Desse total, $7,2 \%$ das mulheres não freqüentaram serviços de pré-natal e $13,8 \%$ dos casos não apresentavam informação sobre pré-natal| ${ }^{(18)}$.

Faz-se necessária uma política conjunta das três esferas do poder público comprometida, verdadeiramente, com melhores indicadores da saúde materna e perinatal. $\mathrm{Na}$ leitura dos indicadores da pesquisa, fica visível que, à medida que são somadas as variáveis de avaliação de processo do PHPN, os percentuais diminuem, indicando que é a realização do conjunto, e não das atividades isoladas, o maior desafio na implementação de uma atenção pré-natal satisfatória.

É da competência da Secretaria de Saúde do Estado em articulação com as secretarias municipais de saúde definir um sistema estadual/regional de assistência obstétrica e neonatal que contemple ações voltadas para a atenção básica, atenção ambulatorial especializada, bem como a assistência hospitalar obstétrica e neonatal de referência para diagnóstico, a atenção ambulatorial à gestação de alto risco e a assistência ao parto de baixo e alto risco.

Às secretarias municipais de saúde compete controlar o cadastro de suas gestantes, garantir assistência prénatal e puerperal, segundo as recomendações do PHPN e as necessidades das gestantes, garantir laboratório para a realização dos exames básicos e o acesso aos exames complementares necessários, mediante programação pactuada integrada regional.

Portanto, é dever do Estado e dos municípios, amparados pelo Governo Federal, a implementação de uma assistência pré-natal de qualidade, assegurando uma maternidade segura para mãe e filho.

\section{CONCLUSÃO}

Considerando que vários fatores contribuem para a mortalidade materna, deve-se planejar estratégias eficazes com enfoque abrangente, baseado em um modelo de atenção primária de saúde ${ }^{(19)}$. O SISPRENATAL, apesar de apresentar indicadores de saúde importantes para analisar a assistência pré-natal prestada, também possui suas limitações. Depende basicamente do que é informado pelos médicos ou enfermeiros, os quais registram mensal- mente na Ficha de Registro Diário do Atendimento à Gestante as consultas realizadas, os exames laboratoriais referentes ao primeiro e terceiro trimestres gestacionais, o anti-HIV e a dose imunizante da vacina antitetânica, bem como a consulta puerperal. Caso os dados não estejam sendo repassados de forma criteriosa à Secretaria de Saúde do Município, os resultados disponíveis podem não ser fidedignos por estarem subestimados. É necessário, pois, a capacitação de profissionais e demais funcionários envolvidos com o SISPRENATAL, com o intuito de gerar alimentação e monitorização adequadas do sistema acerca do acompanhamento pré-natal.

Os dados do Sistema de Informação de Mortalidade (SIM) ${ }^{(20)}$ evidenciam que no período de 2000 a 2005 não houve óbito materno no Município de Quixadá. Quanto à mortalidade perinatal no ano de 2000-2005, o SIM registra, ainda, que antes da implantação do Programa, em 2000, foram registrados 26 óbitos, enquanto que, em 2005, ocorreram 13 óbitos perinatais. Esta diminuição na ocorrência de óbitos perinatais pode sinalizar que o PHPN possa estar contribuindo para diminuir a mortalidade perinatal no município. Os dados deste estudo, porém não permitem realizar tal assertiva.

Sendo o SISPRENATAL um sistema descentralizado, todo gestor, em seu município, utilizando as informações geradas, poderá perceber os avanços e os desafios do PHPN. O monitoramento dos indicadores de processo constitui medida fundamental para melhorar a qualidade dos serviços oferecidos, porque a identificação dos problemas mobiliza para a tomada de decisão e o processo de mudanças. Entretanto, a força fundamental para a concretização das melhorias está no compromisso político com as premissas do PHPN e, principalmente, com as gestantes.

Apesar dos obstáculos a serem suplantados quanto à alimentação e monitorização do SISPRENATAL, este proporciona oportunidades para gestores, profissionais de saúde e usuárias analisarem a assistência prestada às gestantes. Os profissionais de saúde, na função de cuidadores, devem refletir acerca da humanização da assistência pré-natal, que requer, antes de tudo, o cumprimento dos procedimentos básicos preconizados pelo PHPN. Por outro lado, é imperativo o estabelecimento de um relacionamento de confiança com a clientela para prevenir, detectar e controlar agravos na gestação e no puerpério, garantindo o direito fundamental da mulher no exercício da maternidade segura.

\section{REFERÊNCIAS}

1. Peret FJ. Ginecologia \& obstetrícia. 2a ed. Rio de Janeiro: MEDSI; 2000.

2. Brasil. Ministério da Saúde. Assistência Integral à Saúde da Mulher. Bases de ação programática. Brasília: Centro de Documentação do Ministério da Saúde; 1984. 
3. Brasil. Ministério da Saúde. Comitês de Mortalidade Materna. Manual. 2a ed. Brasília; 2002.

4. Matias AO, Moura ERF, Diógenes, MAR, Almeida, MI. Perfil da mortalidade materna nos municípios da 8a. Célula Regional de Saúde do Ceará-Quixadá. In: Therrien SMN, Almeida MI, organizadores. Temas em saúde da família: práticas e pesquisas. Fortaleza: UECE; 2005. p. $21-40$.

5. Ceara. Secretaria da Saúde do Estado. Informe epidemiológico: mortalidade materna. Fortaleza: Núcleo de Epidemiologia; 2003.

6. Brasil. Ministério da Saúde. Secretaria de Atenção à Saúde. Departamento de Ações Programáticas Estratégicas. Pré-natal e puerpério: atenção qualificada e humanizada - manual técnico. Brasília; 2005.

7. Brasil. Ministério da Saúde. Portaria MS/GM n. 569, de 19 de junho de 2000. Institui o Programa de Humanização no Pré-natal e Nascimento, no âmbito do Sistema Único de Saúde - SUS. Diário Oficial da União, Brasília, 18 ago. 2000. Seção 1, p. 112-4.

8. Brasil. Ministério da Saúde. Programa de Humanização no Pré-natal e Nascimento. Informações para gestores e técnicos. Brasília; 2002.

9. Salomon DV. Como fazer uma monografia. 2a ed. São Paulo: Martins Fontes; 1991.

10. Conselho Nacional de Saúde. Resolução n. 196, de 10 de outubro de 1996. Dispõe sobre diretrizes e normas regulamentadora de pesquisas envolvendo seres humanos. Bioética. 1996;4(2 Supl):15-25.

11. Serruya SJ, Lago TG, Cecatti JG. Avaliação preliminar do Programa de Humanização no Pré-natal e Nascimento no Brasil. Rev Bras Ginecol Obstet. 2004;26(7):517-25.

12. Coimbra LC, Silva AAM, Mochel EG, Alves MTSS, Ribeiro VS, Aragão VMF, et al. Fatores associados à inadequação do uso da assistência pré-natal. Rev Saúde Pública. $2003 ; 37(4): 456-62$.
13. Coutinho T, Teixeira MTB, Dain S, Sayd JD, Coutinho LM. Adequação do processo de assistência pré-natal entre as usuárias do Sistema Único de Saúde em Juiz de Fora - MG. Rev Bras Ginecol Obstet. 2003;25(1):717-24.

14. Spindola T, Penna LHG, Progianti JM. Perfil epidemiológico de mulheres atendidas na consulta do prénatal de um hospital universitário. Rev Esc Enferm USP. 2006;40(3):381-8.

15. Rodrigues DP. Representação social de puérperas sobre o cuidado de enfermagem recebido no ciclo gravídico-puerperal [tese]. Fortaleza: Faculdade de Farmácia, Odontologia e Enfermagem, Universidade Federal do Ceará; 2005.

16. Serruya JS, Cecatti JG, Lago TG. O Programa de Humanização no Pré-natal e Nascimento do Ministério da Saúde no Brasil: resultados iniciais. Cad Saúde Pública. 2004;20(5)1281-9.

17. Sousa Júnior PRB, Szwarcwald CL, Barbosa Júnior $A$, Carvalho MF, Castilho EA. Infecção pelo HIV durante a gestação: estudo - sentinela parturiente - Brasil 2002. Rev Saúde Pública. 2004;38(6):764-72.

18. Cavalcante MS, Ramos Junior NA, Silva TMJ, Pontes LRSK. Transmissão vertical do HIV em Fortaleza: revelando a situação epidemiológica em uma capital do Nordeste. Rev Bras Ginecol Obst. 2004;26(2):131-8.

19. Gomes FA, Nakano MAS, Almeida AM, Matuo YK. Mortalidade materna na perspectiva do familiar. Rev Esc Enferm USP. 2006;40(1):50-6.

20. Ceará. Secretaria da Saúde do Estado. Setor de Vigilância Epidemiológica. Sistema de Informação de Mortalidade (SIM). Fortaleza; 2007. 